IV. Sozialrechtliche Fragen 


\title{
Sozialversicherungsrechtliche Fragen bei der Inanspruchnahme von Freizügigkeit
}

\author{
Richard Giesen
}

A. Europäische Sozialrechtskoordinierung und europäische

Sozialrechtsangleichung

I. Regelungen zur europäischen Sozialrechtskoordinierung 175

II. Kurzer Blick auf die Regelungen zur europäischen Sozialrechtsangleichung 176

B. Grundstrukturen der europäischen Sozialrechtskoordinierung 178

I. Einheitliche Kollisionsregeln 178

II. Äquivalenzregeln, insbesondere zur Wartezeitanrechnung 178

III. Leistungsexport $\quad 179$

IV. Fortbestand alter polnisch-deutscher Sozialversicherungsabkommen $\quad 180$

C. Internationale Bestimmung des anwendbaren Rechts 181

I. Grundsatz der ausschließlichen Zuständigkeit eines Staates 181

II. Der Tätigkeitsort als Anknüpfungspunkt für die Bestimmung der
Versicherungspflicht

III. Entsendung 182

$\begin{array}{ll}\text { D. Schluss } & 184\end{array}$

\section{A. Europäische Sozialrechtskoordinierung und europäische} Sozialrechtsangleichung

\section{Regelungen zur europäischen Sozialrechtskoordinierung}

In der EU besteht bereits seit ihrer Gründung als Europäische Wirtschaftsgemeinschaft ein einheitliches System der sozialrechtlichen Koordinierung. Dieses war zunächst Ende 1958 in den EWG-Verordnungen Nr. 3 und Nr. $4^{1}$ geregelt worden, und wurde dann Anfang der 70er Jahre in den EWG-Verordnungen Nr. 1408/71 und Nr.

1 Verordnung Nr. 3 des Rates über die soziale Sicherheit der Wanderarbeitnehmer vom 25.9.1958, AB1. 1958, S. 561; Verordnung Nr. 4 des Rates zur Durchführung und Ergänzung der VO Nr. 3 vom 3.12.1958, AB1. 1958, S. 597. 
574/72 normiert. ${ }^{2}$ Seit dem 1.5.2010 gelten nun die VO 883/2004/EG und ihre Durchführungsverordnung, die VO 987/2009/EG. ${ }^{3}$ Trotz vielfältiger Änderungen und Modifizierungen haben alle drei Verordnungsgenerationen ein einheitliches Regelungssystem und - im Grundsatz - auch ein einheitliches Regelungsziel. Anfangs ging es darum, nur den in den Mitgliedstaaten sozialversicherten Arbeitnehmern keinen Nachteil daraus erwachsen zu lassen, dass sie in Ausübung ihres Freizügigkeitsrechtes (heute Art. 48 AEUV) ihren Wohn- und/oder Beschäftigungsort wechseln. Das ist weiterhin das primäre Ziel, aber heute sind zusätzlich auch die Selbständigen, ${ }^{4}$ Beamten ${ }^{5}$ und Studenten 6 in das Regelungssystem integriert. Des Weiteren besteht ein Mindestmaß an sozialer Sicherung für Touristen sowie für diejenigen, die sich gerade zwecks Nachfrage von Gesundheitsleistungen in einen anderen EU-Mitgliedstaat begeben.

\section{Kurzer Blick auf die Regelungen zur europäischen Sozialrechtsangleichung}

Seit Anbeginn ist europäisches Sozialversicherungsrecht tatsächlich „nur“ Koordinierung und nicht Rechtsangleichung. Im Gegensatz etwa zum Arbeitsrecht sind im Sozialversicherungsrecht nur wenige Regelungen angeglichen worden. Ich möchte hier lediglich zwei Bereiche nennen, nämlich den Schutz des nicht (vollständig) bezahlten Arbeitnehmers bei Insolvenz des Arbeitgebers und das Recht der Diskriminierungsbekämpfung innerhalb der Sozialversicherung.

- Die heutige Richtlinie 2008/94/EG verpflichtet die Mitgliedstaaten, Garantieeinrichtungen zu schaffen, mit deren Hilfe Arbeitnehmer, die aufgrund Zahlungsunfähigkeit des Arbeitgebers nicht oder nicht vollständig bezahlt wurden, Entgeltersatzleistungen erhalten. ${ }^{7}$ Ihre Vorgängerrichtlinie hat seinerzeit für einige Aufmerksamkeit gesorgt, weil der EuGH anlässlich ihrer mangelhaften Umsetzung

2 Verordnung (EWG) Nr. 1408/71 über die Anwendung der Systeme der sozialen Sicherheit auf Arbeitnehmer und Selbständige sowie deren Familienangehörige, die innerhalb der Gemeinschaft zuund abwandern vom 14.6.1971, ABl. L 1971, 149/2; Verordnung (EWG) Nr. 574/72 über die Durchführung der Verordnung (EWG) Nr. 1408/71 über die Anwendung der Systeme der sozialen Sicherheit auf Arbeitnehmer und Selbständige sowie deren Familienangehörige, die innerhalb der Gemeinschaft zu- und abwandern vom 21.3.1972, ABl. L 1972, 74/1.

3 Verordnung (EG) 883/2004 des Europäischen Parlaments und des Rates vom 29.4.2004 zur Koordinierung der Systeme der sozialen Sicherheit, ABl. L 2004, 166/1; L 2004, 204/30; Verordnung (EG) 987/2009 zur Festlegung der Modalitäten für die Durchführung der Verordnung (EG) Nr. 883/2004 über die Koordinierung der Systeme der sozialen Sicherheit vom 16.9.2009, ABl. L 2009, 284/1. S. zur historischen Entwicklung Fuchs in: ders. (Hrsg.), Europäisches Sozialrecht, Kommentar, 5. Aufl. 2010, Einführung, Rn. 7 ff.

4 Verordnung (EWG) 1390/81 vom 29.5.1981, ABl. L 1981, 143/1.

5 Verordnung (EWG) 1608/98 vom 29.6.1998, ABl. L 1998, 209/1.

6 Verordnung (EG) 307/1999 vom 8.2.1999, ABl. L 1981, 038/1.

7 Richtlinie 2008/94/EG vom 22.10.2008 über den Schutz des Arbeitnehmers bei Zahlungsunfähigkeit des Arbeitgebers, AB1. L 2008, 283/36. 
die Grundsätze zur Staatshaftung wegen unzureichender Richtlinienumsetzung entwickelt hat. 8

- Die Regelungen über die Diskriminierungsbekämpfung innerhalb der Sozialversicherung betreffen nicht nur die Gleichbehandlung der Nationalitäten, sondern auch um die im Arbeitsrecht heftig diskutierte Gleichbehandlung der Geschlechter. Letztere wird im Sozialrecht europarechtlich durch die Richtlinie 79/7 zur schrittweisen Verwirklichung des Grundsatzes der Gleichbehandlung von Männern und Frauen im Bereich der sozialen Sicherheit umgesetzt. ${ }^{9}$ Dabei ist zu beachten, dass gemäß Art. 7 Abs. 1 lit. a Richtlinie 79/7 die Festsetzung unterschiedlicher Rentenalter für Männer und Frauen ausdrücklich erlaubt ist. 10

Nach Art. 153 Abs. 1 lit. c AEUV hat die Union zwar eine Regelungskompetenz im Bereich der sozialen Sicherheit. Diese ist aber streng ausgestaltet, denn gemäß Art. 153 Abs. 2 Unterabsatz 3 AEUV wird für darauf beruhende Regelungen ein Einstimmigkeitserfordernis bei der Abstimmung im Rat gefordert - das bedeutet, dass sich alle Staaten über entsprechende Regelungen einig sein müssen.

8 Die Richtlinie war zunächst in manchen Staaten nicht fristgerecht umgesetzt worden, vgl. EuGH 8.11.1990, Rs. 53/88 (Kommission/Griechenland), Slg. 1990 I, 3917 (3932 ff.); EuGH 2.2.1989, Rs. 22/87 (Kommission/Italien), Slg. 1989, 143 (164 ff.). In seiner Entscheidung „Francovich I“ erklärte der EuGH entsprechende Umsetzungsversäumnisse der Mitgliedstaaten für schadensersatzpflichtig, EuGH 19.11.1991, Rs. 6, 9/90 (Francovich I), Slg. 1991, I-5357 (5413 ff.), bestätigt in EuGH 16.12.1993, Rs. C-334/92 (Wagner Miret), Slg. 1993, I-6911 (6931 ff.); vgl. Steiner, European Law Review 18 (1993), 3 ff.; Böhm, JZ 1997, 453 ff. Anzumerken bleibt, dass Herr Francovich, da er persönlich die Anwendungsvoraussetzungen der Richtlinie nicht erfüllte, nie Schadensersatz erhalten hat, s. EuGH 9.11.1995, Rs. C-479/93 (Francovich II), Slg. 1995, I-3843 (3869 ff.); bestätigt in EuGH 10.7.1997, Rs. C-373/95 (Maso), Slg. 1997, I-4051 (4076). Außerdem hat der EuGH 1997 zwei Urteile zum Inhalt der Richtlinie erlassen, aus denen sich die nur unvollständige Umsetzung der Richtlinie auch in Deutschland ergibt, EuGH 10.7.1997, Rs. C-94, 95/95 (Bonifaci), Slg. 1997, I-3969 (4017 ff.); EuGH 10.7.1997, Rs. C-373/95 (Maso), Slg. 1997, I-4051 (4077 f.); vgl. hierzu Krause, ZIP 1998, 56 ff.

9 Richtlinie 79/7 vom 19.12.1978, ABl. L 1979, 6/24.

10 EuGH 7.7.1992, Rs. C-9/91 (Equal Opportunities Commission), Slg. 1992, I-4297 (4337 ff.); EuGH 30.3.1993, Rs. C-328/91 (Thomas), Slg. 1993, I-1247 (1271 ff.); EuGH 1.7.1993, Rs. C-154/92 (van Cant), Slg. 1993, I-3811 (3834 f.); EuGH 19.10.1995, Rs. C-137/94 (Richardson), Slg. 1995, I-3407 (3430 ff.); vgl. EuGH 16.2.1982, Rs. 19/81 (Burton), Slg. 1982, I-555 (576); EuGH 30.1.1997, Rs. C-139/95 (Balestra), Slg. 1997, I-549 (577 ff.). Laut EuGH erfasst diese Zulassung der Ungleichbehandlung nicht nur die Altersrente, sondern auch (mit der Altersrente übereinstimmende) Altersregelungen in der Invaliditätsversicherung, EuGH 11.8.1995, Rs. C-92/94 (Graham), Slg. 1995, I-2521 (2552 ff.). Andererseits hat der EuGH in mittlerweile ständiger Rechtsprechung die Begünstigung von Frauen in Frühverrentungsregeln der betrieblichen Altersversorgung wegen Geschlechterdiskriminierung (Art. 157 AEUV, früher Art. 141 EG-Vertrag) für unzulässig erklärt, und zwar mit Wirkung vom 17.5.1990, s. EuGH 17.5.1990, Rs. C-262/88 (Barber), Slg. 1990, I-1889 ff.; EuGH 9.10.2001, Rs. C-379/99 (Menauer), Slg. 2001, I-7275, Rn. 17 ff.; zu alledem Schlachter in: Erfurter Kommentar zum Arbeitsrecht, 12. Aufl. 2012, Art. 157 AEUV, Rn. 5; Tillmanns, in: Henssler/Willemsen/Kalb (Hrsg.), Arbeitsrecht Kommentar, 4. Aufl. 2010, Art. 157 AEUV, Rn. 11 ff. 


\section{B. Grundstrukturen der europäischen Sozialrechtskoordinierung}

Damit stellt sich die Frage, wie die Europäische Sozialrechtskoordinierung funktioniert. Die VO 883/2004/EG und ihre Durchführungsverordnung VO 987/2009/EG verfolgen - ebenso wie ihre Vorgängerregelungen ${ }^{11}$ - ein einheitliches Regelungsprinzip, das über drei Grundelemente verfügt, nämlich einheitliche Kollisionsregeln, Äquivalenzregeln und Vorschriften zum Leistungsexport (dazu sogleich unter 1. bis 3.). Für polnisch-deutsche Rentenbiographien sind dabei die Sonderregelungen zum teilweisen Fortbestand älterer bilateraler Sozialversicherungsabkommen zu beachten (dazu unter 4.).

\section{Einheitliche Kollisionsregeln}

Die Art. 11 ff. VO 883/2004/EG enthalten für alle Mitgliedstaaten einheitliche Kollisionsregeln. Diese bewirken durch gleichförmige Anknüpfung, die grundsätzlich auf den Beschäftigungsort abstellt, dass für jeden Sozialversicherten jeweils nicht mehr und nicht weniger als ein Staat zuständig ist. Auf diese Weise werden Doppelversicherungen (mit vor allem doppelten Beitragspflichten) ebenso ausgeschlossen wie Versicherungslücken. Schulbeispiel ist der Fall des Arbeitnehmers, der in Staat A arbeitet und in Staat B wohnt. Wenn für die Krankenversicherung in Staat A das Beschäftigungsortsprinzip gelten würde und in Staat B das Wohnortsprinzip, wäre der Arbeitnehmer doppelt krankenversichert. Wenn umgekehrt in Staat B das Beschäftigungsortsprinzip und in Staat A das Wohnortsprinzip gelten würde, wäre der Arbeitnehmer gar nicht krankenversichert. Beides soll nicht sein und wird durch einheitliche Anknüpfung verhindert. Die Einzelheiten werden unter III. näher dargelegt.

\section{II. Äquivalenzregeln, insbesondere zur Wartezeitanrechnung}

Des Weiteren existieren in den Verordnungen einige Äquivalenzregeln, welche die Verwirklichung bestimmter Tatbestände im Ausland mit der Verwirklichung im Inland gleichstellen. Neben der allgemeinen Regel des Art. 5 VO 883/2004 ist die wohl wichtigste Äquivalenzregel diejenige des Art. 6 VO 883/2004. Sie sieht vor, dass bei der Erfüllung von Wartezeiten für Sozialleistungsansprüche auch Auslandszeiten zu berücksichtigen sind. Schulbeispiel ist hier der Fall des Arbeitnehmers, der acht Jahre im europäischen Ausland gearbeitet hat und dann drei Jahre in Deutschland. Wenn er das Rentenalter erreicht, hat er nach deutschem Recht allein keinen Anspruch auf Altersrente, weil er die in $\S \S 35,50$ SGB VI geforderten fünf Jahre Wartezeit noch nicht erfüllt

11 S. oben Fußn. 1, 2. 
hat. Da aber für die Wartezeitregelung auch Auslandszeiten zu berücksichtigen sind, erwirbt er seinen Anspruch mithilfe der im EU/EWR-Ausland zurückgelegten Versicherungszeiten. Freilich geht dies nicht so weit, dass auch Entgeltpunkte für die ausländischen Zeiten gewährt würden. Für den Anspruchserwerb greift damit Äquivalenz, nicht aber für die Anspruchshöhe. Hierfür müssen Anwartschaften aus jedem Staat für die dort zurückgelegten Jahre gesondert erworben werden. Es ist klar, dass Art. 6 VO 883/2004 nicht nur für die Altersvorsorge, sondern beispielsweise auch für den Erwerb von Arbeitslosengeldansprüchen und Invaliditätsrentenansprüchen bedeutsam ist.

\section{Leistungsexport}

Zudem verpflichten die Verordnungen die Sozialversicherungsträger zum Leistungsexport.

- Das ist für Geldleistungen eine relativ unproblematische Angelegenheit. Der eben genannte Rentner, der aus zwei unterschiedlichen Staaten Renten beanspruchen kann, erhält diese Renten auch dann ausgezahlt, wenn er sich nicht auf deren Territorium aufhält. Kehrt er also beispielsweise im Alter in das Land zurück, in dem er aufgewachsen ist, aber nie gearbeitet hat, erhält er dennoch Altersversorgung. Geldleistungen sind nicht immer nur Renten, sondern beispielsweise auch Krankengeld und - nach der Rechtsprechung des EuGH - auch die vom Arbeitgeber geschuldete Entgeltfortzahlung im Krankheitsfall. Auch hier muss also geleistet werden, unabhängig davon, ob sich der kranke Arbeitnehmer im Inland oder im Ausland aufhält. ${ }^{12}$

- Bei Sachleistungen dagegen lässt sich der Leistungsexport nicht so leicht durchführen. Um ihn zu ermöglichen, behilft man sich mit dem sogenannten Prinzip der aushelfenden Sachleistungsgewährung. Dieses Prinzip besagt, dass statt des zuständigen Trägers die Träger im jeweiligen Wohn- oder Aufenthaltsstaat des Leistungsberechtigten nach ihren Rechtsvorschriften, aber für den zuständigen Träger und auf seine Kosten tätig werden. Der Versicherte bezieht außerhalb des zuständigen Staates also zwar Leistungen, aber nicht aus den Händen des zuständigen Trägers, sondern vom ausländischen Träger vor Ort nach dessen Recht. Der ausländische Träger erhält dann seine Aufwendungen vom zuständigen Trä-

12 S. Art. 21 VO 883/2004/EG. Vgl. zur Entgeltfortzahlung im Krankheitsfall EuGH 3.6.1992, Rs. C45/90 (Paletta I), Slg. 1992, I-3423 (3462 ff.); EuGH 2.5.1996, Rs. C-206/94 (Paletta II), Slg. 1996, I-2357 (2389 ff.). Das Vorliegen einer EU/EWR-Arbeitsunfähigkeitsbescheinigung nach Art. 18 VO 574/72 (s. heute Art. 5, 27 VO 987/2009) bewirkt demnach gemäß EuGH „Paletta I“ und „Paletta II" die Annahme, dass der Arbeitnehmer tatsächlich krank war. Diese Annahme kann der Arbeitgeber (und ebenso ein anderer Träger) gemäß „Paletta II“ dadurch widerlegen, dass er Nachweise erbringt, aus denen sich die missbräuchliche oder betrügerische Vorlage der Bescheinigung ergibt. Vgl. zu alledem Abele, NZA 1996, 631 f.; Schlachter, EuZW 1996, 377 f.; Heinze/Giesen, BB 1996, $1830 \mathrm{ff}$. 
ger - u.U. als pauschale Summe - ersetzt, soweit zwischen den Staaten kein entsprechendes Verzichtsübereinkommen besteht. ${ }^{13}$

\section{Fortbestand alter polnisch-deutscher Sozialversicherungsabkommen}

Bei alledem bereiten die alten Sozialversicherungsabkommen zwischen Polen und Deutschland auch noch unter Geltung der VO 883/2004 Sorgen. Für Altfälle finden sie im Bereich der Rentenversicherung weiterhin teilweise Anwendung. ${ }^{14}$ Nach den Abkommen gilt bei der Gewährung von Renten teils das so genannte Eingliederungsprinzip. Dieses besagt, dass Renten jeweils vom Wohnstaat zu gewähren sind, und zwar so, als seien sämtliche Versicherungs-, Beschäftigungs- und sonstige Zeiten dort zurückgelegt worden. Dieses Prinzip hat in den Zeiten endgültiger Umsiedlung durch den ,eisernen Vorhang" sicher seine Berechtigung gehabt. Heute bereitet es für die betroffenen älteren Menschen dagegen erhebliche Schwierigkeiten. Wenn diese etwa aus familiären Gründen oder zwecks Wahrnehmung besserer Pflegemöglichkeiten von Deutschland nach Polen zurückwandern, erleiden sie erhebliche Renteneinbußen. Diese Renteneinbußen sind den Betroffenen teils nicht bekannt. Sie werden - soweit ersichtlich - bei Zurückwanderung nach Deutschland auch nicht rückgängig gemacht.

Letzteres weckt einige rechtliche Zweifel. Zu den betreffenden Fragestellungen sei auf die Beiträge von Bogdan Borecki und Stephan Fasshauer verwiesen. ${ }^{15}$

Soweit zu den Grundstrukturen der Sozialrechtskoordinierung. Lassen Sie mich nun zu einem Schwerpunkt der Regelung der VO (EG) 883/2004 kommen, nämlich dem Kollisionsrecht, also der Regelung des anwendbaren Rechts.

13 Vgl. Art. 18 Abs. 1 S. 2, 19 Abs. 1 S. 2, 20 Abs. 2 S. 1, 23, 24 Abs. 1 S. 2, 26, 35, 36, 41 VO 883/2004; auf die betreffenden Regelungen wird teils durch andere Vorschriften noch verwiesen, wodurch sich ihr Anwendungsbereich noch erweitert. S. zur aushelfenden Sachleistungsgewährung Bieback, in: Fuchs (Fußn. 3), Vorbem. vor Art. 17 VO 883/2004, Rn. 30 ff.; Fuchs daselbst, Art. 36 VO 883/2004, Rn. 4 ff. S. zur Exportverpflichtung nach Maßgabe der Regelungen über die Dienstleistungsfreiheit den Beitrag von Daniel Lach sowie Bieback, in: Fuchs (Fußn. 13), Rn. 30 ff.

14 Art. 8 Abs. 1 VO 883/2004 i.V.m. Anhang II „Deutschland-Polen“, lit. a, b VO 883/2004; s. auch die Vorgängerregelung Art. 3 Abs. 3, Art. 7 Abs. 2 lit. c VO 1408/71 i.V.m. Anhang III, A Nr. 84 lit. a, b zur VO 1408/71. Die dortigen Verweise betreffen die Abkommen zwischen der DDR und Polen aus den Jahren 1957 und 1988 sowie die Abkommen zwischen der BRD und Polen aus den Jahren 1975 und 1990.

15 In diesem Band, S. 257 ff. und 235 ff. 


\section{Internationale Bestimmung des anwendbaren Rechts}

\section{Grundsatz der ausschließlichen Zuständigkeit eines Staates}

Die Kollisionsregeln der VO 883/2004 bestimmen das jeweilig auf einen Fall anzuwendende nationale Recht und damit gleichzeitig die Zuständigkeit eines nationalen Sozialversicherungsträgers. Die VO 883/2004 folgt dabei - wie auch schon ihre Vorgängerverordnungen - dem Grundsatz der ausschließlichen Zuständigkeit eines Staates. Art. 11 Abs. 1 S. 1 VO 883/2004 stellt ausdrücklich klar, dass Personen, für die die Verordnung gilt, „den Rechtsvorschriften nur eines Mitgliedstaats“ unterliegen können. Das hat nicht nur für die Leistungserbringung die Funktion einheitlicher Zuordnung. Aus der einheitlichen Zuordnung folgt meines Erachtens auch, dass bei Erwerbstätigkeit in unterschiedlichen Mitgliedstaaten für die Beitragserhebung sowie für die Zahlung von Renten, Kranken- und Arbeitslosengeld das gesamte, auch im Ausland erzielte Einkommen berücksichtigt wird. Dies ergibt sich nicht zuletzt aus der auch auf Einkommen bezogenen Äquivalenzregel des Art. 5 lit. b VO 883/2004/EG. ${ }^{16}$ Ein Wahlrecht des Versicherten zwischen verschiedenen Staaten soll aus Gründen der Rechtssicherheit und zur Vermeidung von Missbräuchen und Belastungsgefällen zwischen den Staaten vermieden werden. ${ }^{17}$ Dennoch existieren - allerdings nur sehr vereinzelte - Ausnahmen von der ausschließlichen Zuständigkeit eines Sicherungssystems. ${ }^{18}$

16 Dies ließ sich schon vor Geltung von Art. 5 lit. b VO 883/2004/EG im Umkehrschluss aus der Aussage des EuGH schließen, der im Fall der ausnahmsweisen Doppelversicherung in zwei Mitgliedstaaten nach der früheren Regelung des Art. 14c lit. b VO 1408/71 i.V.m. Anhang VII (s. heute Art. 14 Abs. 3 VO 883/2004/EG) erklärt hat, hier dürften bei der Beitragserhebung nur die im jeweiligen Staat erzielten Einnahmen berücksichtigt werden, EuGH 30.1.1997, Rs. C-340/94 (de Jaeck), Slg. 1997, I-461 (507 f.). Der Grundsatz der ausschließlichen Zuständigkeit eines Staates hat also zur Folge, dass ein Versicherter, der innerhalb eines bestimmten Zeitraums in mehreren Mitgliedstaaten tätig war, in keinem der betreffenden Staaten für Zeiten versicherungs- (und damit auch beitrags-) pflichtig sein kann, die er im anderen Staat verbracht hat, EuGH 5.5.1977, Rs. 102/76 (Perenboom), Slg. 1977, I-815 (821 f.); s. auch EuGH 10.7.1986, Rs. 60/85 (Luijten), Slg. 1986, I-2365 (2372 f.); EuGH 16.1.1992, Rs. C-57/90 (Kommission/Frankreich), Slg. 1991, I-75 (97).

17 Vgl. EuGH 29.6.1988, Rs. 58/87 (Rebmann), Slg. 1988, I-3467 (3485 ff.), wo der Gerichtshof die Annahme eines Wahlrechts bei Rechtsunklarheiten mit Blick auf Art. 51 EGV und das System der VO 1408/71 ausdrücklich ablehnt; s. auch EuGH 12.6.1986, Rs. 1/85 (Miethe), Slg. 1986, I-1837 (1850); EuGH 7.7.1992, Rs. C-102/91 (Knoch), Slg. 1992, I-4341 (4390 f.).

18 Eine Ausnahme, Art. 14 Abs. 2 S. 2 VO 883/2004, betrifft die Konkurrenz mehrerer freiwilliger Versicherungen und die Konkurrenz von Pflichtversicherung und freiwilliger Versicherung. Eine weitere Ausnahme betrifft spezielle Fälle der Konkurrenz von selbständiger und unselbständiger Tätigkeit, die jeweils in unterschiedlichen Mitgliedstaaten ausgeübt werden, Art. 14 Abs. 3 VO 883/2004, vgl. dazu EuGH Rs. de Jaeck (Fußn. 17). 


\section{Der Tätigkeitsort als Anknüpfungspunkt für die Bestimmung der Versicherungspflicht}

Nach der Grundregel des Art. 11 Abs. 3 lit. a VO 883/2004 ist für alle abhängig Beschäftigten und Selbständigen der Tätigkeitsort einschlägiger Anknüpfungspunkt für die Bestimmung ihrer Versicherungspflicht, und zwar auch bei Teilzeitbeschäftigung. ${ }^{19}$ Natürlich bestehen hier ebenfalls Sondervorschriften. Für Beamte gilt beispielsweise das Recht des Staates, dem die ihn beschäftigende Verwaltungseinheit angehört (Art. 11 Abs. 3 lit. b VO 883/2004). Eigene Regelungen gibt es außerdem etwa für Wehr- und Zivildienstleistende, Seeleute und Vertragsbedienstete der EU (Art. 11 Abs. 3 lit. c, Abs. 4, Art. 15 VO 883/2004).

\section{Entsendung}

Besondere Bedeutung haben - gerade für unsere unmittelbar benachbarten Staaten Polen und Deutschland - die Grundsätze zur Entsendung. Bei der Entsendung divergieren der Beschäftigungsort und der Ort des wirtschaftlichen und sozialen Erfolgs. ${ }^{20} \mathrm{Da}-$ bei ist es aus wirtschaftlichen und verwaltungstechnischen Gründen nicht immer sinnvoll, einen Versicherten, welcher nur kurze Zeit im Ausland tätig ist, der dort geltenden Sozialrechtsordnung zu unterwerfen. Mit Blick darauf enthält Art. 12 VO 883/2004 (ähnlich wie $\S \S 4 \mathrm{f}$. SGB IV ${ }^{21}$ ) eine Sonderregelung, gemäß der eine Person, die gewöhnlich in einem bestimmten Mitgliedstaat tätig und demnach dort sozialversichert ist, dies auch bei Auslandsentsendung bleibt, sofern die voraussichtliche Dauer der betreffenden Tätigkeit voraussichtlich nicht 24 Monate übersteigt und - soweit ein Arbeitnehmer und kein Selbständiger betroffen ist - auch kein anderer Arbeitnehmer bei der betreffenden Tätigkeit abgelöst wird.

Für Arbeitnehmer müssen im Einzelnen folgende Voraussetzungen erfüllt sein, um den Entsendetatbestand des Art. 12 Abs. 1 VO 883/2004 zu erfüllen.

- Abordnung des Arbeitnehmers:

Erste Bedingung ist, dass der Arbeitnehmer entsandt wird, sich also zur Ausführung einer Arbeit auf Rechnung seines Arbeitgebers ins Ausland begibt. Nach der Rechtsprechung des EuGH ist eine Entsendung auch dann möglich, wenn die Einstellung nur zum Zweck der Abordnung erfolgt. ${ }^{22}$ Im Falle der grenzüber-

19 EuGH 3.5.1990, Rs. C-2/89 (Kits van Heijningen), Slg. 1990, I-1755 (1773).

20 Eichenhofer, Internationales Sozialrecht, 1994, Rn. $306 \mathrm{ff}$.

21 S. hierzu Steinmeyer, Die Einstrahlung im internationalen Sozialversicherungsrecht; Seewald, in: Kasseler Kommentar, §§ 4, 5 SGB IV; Giesen, VSSR 2008, 327 ff.

22 EuGH 5.12.1967, Rs. 19/67 (Van der Vecht), Slg. 1967, I-461 (472 f.); EuGH 17.12.1970, Rs. 35/70 (Manpower), Slg. 1970, I-1251 (1257); EuGH 10.2.2000, Rs. C.202/97 (Fitzwilliam), Slg. 2000, I-993 ff.; Beschluss Nr. 128 der Verwaltungskommission der EG für die soziale Sicherheit 
schreitenden Arbeitnehmerüberlassung ist ebenfalls eine Entsendung anzunehmen, so dass hier das sozialversicherungspflichtige Beschäftigungsverhältnis beim Verleiher anzusiedeln ist. ${ }^{23}$ Das gilt nur dann nicht, wenn das Zeitarbeitsunternehmen seine Tätigkeit ausschließlich oder überwiegend im Entsendestaat ausübt. ${ }^{24}$

- Fortbestehen des Beschäftigungsverhältnisses:

Weiter muss bei der Entsendung das Beschäftigungsverhältnis fortbestehen, es darf also nicht für die Dauer der Tätigkeit im Ausland unterbrochen worden sein. 25

- Abordnungshöchstdauer:

Die voraussichtliche Dauer der Arbeit im Ausland darf 24 Monate nicht überschreiten. Nach Art. 16 Abs. 1 VO 883/2004 können längere Fristen zugelassen werden. Das wird regelmäßig dann geschehen, wenn Verlängerungen der Abordnungsdauer keine Wettbewerbsnachteile für Unternehmen im Beschäftigungsstaat mit sich bringen. ${ }^{26}$

- Keine Entsendung im Rotationsverfahren:

Außerdem greift die Sonderregel dann nicht, wenn ein Arbeitnehmer einen anderen ablöst, für den die Entsendezeit abgelaufen ist, Art. 12 Abs. 1 VO 883/2004. Hierdurch soll verhindert werden, dass mittels der Entsendung von Arbeitnehmern im Rotationsverfahren für ein und dieselbe Tätigkeit die Anwendung der sozialrechtlichen Vorschriften des Einsatzstaates umgangen wird. ${ }^{27}$

Für Selbständige gilt nach Art. 12 Abs. 2 VO 883/2004 Ähnliches. Allerdings kann hierbei nicht auf die Entsendung im Rahmen des Arbeitsverhältnisses zwischen Entsende- und Gaststaat abgestellt werden, denn dem Selbständigen fehlt eine dem Arbeitsverhältnis vergleichbare fortbestehende rechtliche Verbindung zum Entsendestaat. An die Stelle des Arbeitsverhältnisses tritt daher die vorübergehende Tätigkeit im Ausland unter Beibehaltung einer rein tatsächlichen Bindung an den Entsendestaat. ${ }^{28}$ Die Frage, ob jemand eine „selbständige Tätigkeit“ nach Art. 12 Abs. 2 VO 883/2004 oder eine abhängige Beschäftigung ausübt, richtet sich nach dem jeweiligen nationalen Recht. ${ }^{29}$

Bei alledem ist zu beachten, dass in arbeitsrechtlicher Hinsicht andere Regeln anzuwenden sind. Zwar bleibt es im Fall einer Entsendung nach Maßgabe von Art. 8 Rom I-

der Wanderarbeitnehmer, s. heute auch Beschluss Nr. 128; Steinmeyer, in: Fuchs (Fußn. 3), Art. 12 VO 883/2004, Rn. 12 ff.

23 EuGH Rs. Manpower (Fußn. 22).

24 EuGH Rs. Fitzwilliam (Fußn. 22).

25 Steinmeyer, in: Fuchs (Fußn. 3), Art. 12 VO 883/2004, Rn. 25.

26 Vgl. ebd., Art. 16 VO 883/2004, Rn. 1 ff.

27 Vgl. ebd., Art. 16 VO 883/2004, Rn. 15.

28 Ebd., Art. 16 VO 883/2004, Rn. 20 ff.

29 EuGH 30.1.1997, Rs. C-221/95 (Hervein), Slg. 1997, I-609 (639 ff.); EuGH 20.10.2000, Rs. C242/99 (Vogler), Slg. 2000, I-9083 ff.; vgl. auch EuGH Rs. de Jaeck (Fußn. 17). 
VO (wie im Sozialversicherungsrecht) weiterhin bei der Anwendung des Rechts des Entsendestaates. Jedoch gelten nach den näheren Bestimmungen der Entsendegesetze in Deutschland des Arbeitnehmerentsendegesetzes, AEntG - zusätzlich besondere Mindestschutzregelungen des Einsatzstaates. Solche Sondervorschriften werden durch die Entsenderichtlinie zugelassen. Hierzu sei auf den Beitrag von Ludwik Florek zum Entsenderecht verwiesen. ${ }^{30}$

\section{Schluss}

Die hiesige Darstellung diente als Einführung für die weiteren Beiträge und benötigt deshalb kein verfrühtes Fazit. Mir scheint, dass es der EU prinzipiell - trotz vieler technischer und auch administrativer Schwierigkeiten - gelingt, die unterschiedlichen Sozialrechtsordnungen so zu koordinieren, dass die Inanspruchnahme von Freizügigkeit nicht übermäßig behindert wird. Das schließt das Verhältnis von Polen und Deutschland ein, wenn auch die teils noch fortbestehenden alten Sozialversicherungsabkommen hier Schwierigkeiten bereiten.

30 In diesem Band, S, $161 \mathrm{ff}$. 


\title{
Koordinierung von Alters- und Hinterbliebenenrenten
}

\author{
Krzysztof Ślebzak
}

$\begin{array}{lr}\text { A. Allgemeines } & 185\end{array}$

B. Versicherungsfälle $\quad 186$

C. Europäisierung der Antragstellung auf Rente 187

D. Versicherungszeiten 189

E. Berechnung der Rente 193

I. Autonome Leistung 193

II. Anteilige Leistung 193

III. Höchstbetragsregelung 194

IV. Rentenberechnung bei den Versicherungs- und Wohnzeiten
unter einem Jahr

V. Neuberechnungen und Anpassungen der Leistungen 195

F. Antikumulierungsregelungen 196

\section{A. Allgemeines}

Die Freizügigkeit der Personen innerhalb der Europäischen Union erfordert eine Koordinierung der Systeme der sozialen Sicherheit. Bei der Wahrnehmung der Freizügigkeit können die Personen den Rentensystemen mehrerer Mitgliedstaaten unterliegen. In diesem Fall sind das Europäische Parlament und der Rat verpflichtet, die notwendigen Maßnahmen für die Sicherstellung der Freizügigkeit der Arbeitnehmer vorzunehmen, insbesondere ein System einzuführen, das den zu- und abwandernden Arbeitnehmern und Selbstständigen sowie deren anspruchsberechtigten Angehörigen und den Personen, die in den Hoheitsgebieten der Mitgliedstaaten wohnen, die Anrechnung aller nach den verschiedenen innerstaatlichen Rechtsvorschriften berücksichtigten Zeiten für den Erwerb, die Berechnung und die Aufrechterhaltung des Leistungsanspruchs sichert (Art. 48 Abs. 1 lit. a) AEVV). Somit müssen die eventuellen Nachteile, die bei der Wahrnehmung der Freizügigkeit entstehen, ausgeglichen werden.

Die rechtliche Problematik von Alters- und Hinterbliebenenrenten wurde im Kapitel 5 der VO 883/2004, das das Kapitel 3 des Titels III der VO 1408/71 ersetzt, geregelt. Die Vorschriften betreffen neben den Rentenleistungen bei Alter und Tod auch die Leis- 